\title{
Reação liquenóide de contato associada a restauração de amálgama: relato de caso
}

\author{
Lichenoid contact reaction associated with amalgam restoration: case report
}

Liquenoide recuento relacionado con la restauración de amalgama: relato de caso

Jessika Nathalia Victor Lucas ${ }^{1 *}$, Leonara Severiano e Silva ${ }^{1}$, Vanessa Pereira Gonçalves de Souza ${ }^{1}$, Danielly Melo Gomes ${ }^{1}$, José de Castro Jatobá Neto ${ }^{1}$, Lídia Maria de Souza Lisboa ${ }^{1}$, Natália Leão Gonçalves ${ }^{1}$, Andreza Pereira Gonçalves de Souza ${ }^{3}$, Kattyélen Luzyane de Farias ${ }^{1}$, Jysllene Nataly Victor Lucas ${ }^{1}$, Yuri Jivago Tenório Costa Lôbo Peixoto ${ }^{1}$, Vanessa de Carla Batista dos Santos ${ }^{2}$.

\section{RESUMO}

Introdução: A reação liquenóide oral (RLO) é uma alteração patológica, de origem inflamatório-crônica e muco-cutânea, que apresenta caráter imunológico determinado através do contato com variadas substâncias exógenas. Essa reação manifesta-se através de reações de hipersensibilidade, na grande maioria das vezes por materiais odontológicos, tendo como fator principal o amálgama dental. Relato de Caso: O presente trabalho relata o caso de uma paciente do sexo feminino que tem 32 anos, feoderma, que compareceu a clínica queixando-se de dormência e dor em uma lesão na língua, há aproximadamente três meses e sem motivo aparente. As reações liquenóides orais tem origem a partir de um episódio de hipersensibilidade associado a materiais odontológicos, e apresentam coloração branca. Quanto a sua localização, a reação liquenóide acomete a região posterior da mucosa jugal, superfície ventral das bordas laterais da língua, língua propriamente dita e gengiva e evidencia a predileção da lesão pelo aparecimento em mulheres. Existe a possibilidade do aparecimento de sintomas, tais como dor e ardência. No presente caso o diagnóstico foi realizado associando os critérios clínicos e histopatológicos da lesão e o tratamento da sintomatologia dolorosa foi prescrito o uso de algumas medicações e após o tratamento sintomatológico, foi realizada a troca das restaurações de amálgama pela resina composta. Conclusão: Sendo assim faz-se necessário o conhecimento das características clínicas e histopatológicas das lesões liquenóides orais, para realização de um diagnóstico preciso e determinação do tratamento a ser realizado, embora ainda exista uma carência de informações quanto a melhor forma de diagnóstico.

Palavras-chave: Patologia, Hipersensibilidade, Mucosa Bucal.

\begin{abstract}
Introduction: The oral lichenoid reaction (RLO) is a pathological alteration, of inflammatory-chronic origin and muco-cutaneous, that presents immunological character determined by the contact with various exogenous substances. This reaction manifests itself through hypersensitivity reactions, most of the time by dental materials, with dental amalgam as its main factor. Case Report: The present study reports the case of a female patient who is 32 years old, feoderma, who attended the clinic complaining of numbness and pain in a lesion on the tongue, for approximately three months and for no apparent reason. Oral lichenoid

${ }^{1}$ Discente do curso de odontologia do Centro Universitário Cesmac. * E-mail: ihenathalia@hotmail.com

2 Docente do curso de odontologia do Centro Universitário Cesmac.

${ }^{3}$ Cirurgiã-dentista.
\end{abstract}

SUBMETIDO EM: 11/2018

ACEITO EM: 12/2018

PUBLICADO EM: 02/2019 
reactions originate from a hypersensitivity episode associated with dental materials, and are white in color. As for its location, the lichenoid reaction affects the posterior region of the jugal mucosa, the ventral surface of the lateral borders of the tongue, tongue itself and gingiva and evidences the predilection of the lesion for appearance in women. There is the possibility of the onset of symptoms, such as pain and burning. In the present case the diagnosis was made associating the clinical and histopathological criteria of the lesion and the treatment of the painful symptomatology was prescribed the use of some medications and after the symptomatic treatment, the amalgam restorations were replaced by the composite resin. Conclusion: Thus, it is necessary to know the clinical and histopathological features of oral lichenoid lesions, in order to carry out an accurate diagnosis and determination of the treatment to be performed, although there is still a lack of information regarding the best form of diagnosis.

Keywords: Pathology, Hypersensitivity, Mouth Mucosa.

\section{RESUMEN}

Introducción: La reacción líquenóide oral $(R L O)$ es una alteración patológica, de origen inflamatorio-crónico y muco-cutáneo, que presenta carácter inmunológico determinado a través del contacto con variadas sustancias exógenas. Esta reacción se manifiesta a través de reacciones de hipersensibilidad, en la gran mayoría de las veces por materiales odontológicos, teniendo como factor principal la amalgama dental. Relato de Caso: El presente trabajo relata el caso de una paciente femenina que tiene 32 años, feoderma, que asistió a la clínica quejándose de adormecimiento y dolor en una lesión en la lengua, hace aproximadamente tres meses y sin motivo aparente. Las reacciones liquenóides orales proceden de un episodio de hipersensibilidad asociado a materiales odontológicos, y presentan coloración blanca. En cuanto a su localización, la reacción liquenóide acomete la región posterior de la mucosa jugal, superficie ventral de los bordes laterales de la lengua, lengua propiamente dicha y encía y evidencia la predilección de la lesión por la aparición en mujeres. Existe la posibilidad de la aparición de síntomas, tales como dolor y ardor. En el presente caso el diagnóstico fue realizado asociando los criterios clínicos e histopatológicos de la lesión y el tratamiento de la sintomatología dolorosa fue prescrito el uso de algunas medicaciones y después del tratamiento sintomatológico, se realizó el cambio de las restauraciones de amalgama por la resina compuesta. Conclusión: Por lo tanto, se hace necesario el conocimiento de las características clínicas e histopatológicas de las lesiones liquenóides orales, para realizar un diagnóstico preciso y determinación del tratamiento a realizar, aunque todavía existe una carencia de informaciones como la mejor forma de diagnóstico.

Palabra-clave: Patologia, Hipersensibilidad, Mucosa Bucal.

\section{INTRODUÇÃO}

A reação liquenóide oral (RLO) é uma alteração patológica, de origem inflamatório-crônica e mucocutânea, que apresenta caráter imunológico determinado através do contato com variadas substâncias exógenas. Essa reação manifesta-se através de reações de hipersensibilidade, na grande maioria das vezes por materiais odontológicos, tendo como fator principal o amálgama dental (BERNARDES et al., 2007; SOARES-SANTOS et al., 2017).

São conhecidas quatro tipos de reações liquenóides orais, segregadas de acordo com o seu fator desencadeante e classificadas em: reação liquenóide de contato, caracterizada por uma hipersensibilidade ao material odontológico empregado em restaurações, principalmente materiais metálicos; reação liquenóide associada a drogas, caracterizada pela melhora ou desaparecimento das estrias esbranquiçadas após a troca ou retirada do medicamento desencadeante; reação liquenóide por doença do enxerto contra o hospedeiro, que é uma reação imunológica entre as células linfoides do doador e os tecidos do hospedeiro e a reação liquenóide não classificada que não apresenta características das outras e aparece simétrica e 
bilateralmente (ARTICO et al., 2011; LUIZ., 2007; BARROS et al. 2015; ROZZA et al., 2007; WERNECK, MIRANDA, JÚNIOR, 2016).

Clinicamente caracteriza-se pelo aparecimento de lesões no local de contato com o alergeno. Quando em mucosa, tem preferência pelas áreas de gengiva, língua e mucosa jugal e apresentam fator causal de desencadeamento de resposta imunológica conhecida (LUIZ. 2007). Geralmente, são assintomáticas, mas quando relatado a presença de sintomas, o paciente descreve a sensação de gosto metálico, prurido, ardência e dor. Clinicamente, as lesões associadas à reação liquenóide apresentam-se através de estrias reticulares, de forma eritematosa, ulcerativa, erosiva ou em vesículas, e de coloração esbranquiçada (BERNARDES., et al 2007; SOARES- SANTOS et al., 2017).

As Reação Liquenóide de Contato (RLC) apresentam dificuldade no diagnostico final em virtude da semelhança com outras alterações da mucosa, e tem como diagnóstico diferencial o líquen plano oral que apresenta características clinicas indistintas, mas localizações incomuns podem determinar o seu diagnóstico, visto que histologicamente a reação liquenóide e o líquen plano são indistinguíveis. Dessa forma o diagnóstico da RLC não pode ser finalizado baseado apenas em características isoladas e sim em associação do aspecto clínico com os critérios histopatológicos (ARTICO et al., 2011; LUIZ. 2017; WERNECK, MIRANDA, JÚNIOR, 2016).

Histologicamente, apresenta características bem peculiares, com presença de infiltrado inflamatório profundo e caracteristicamente, existe um misto de células inflamatórias como os linfócitos plasmócitos, neutrófilos ou eosinófilos (WERNECK, MIRANDA, JÚNIOR, 2016).

A melhor forma de tratamento para a reação liquenóide de contato é a remoção do fator desencadeante do processo de hipersensibilidade, como a remoção de restaurações metálicas ou outro material restaurador, favorecendo a diminuição ou o desaparecimento da lesão e em casos de sintomatologia, podese recorrer à terapia medicamentosa com analgésicos para diminuição da dor e anti-inflamatórios para a diminuição da inflamação associada. Em geral, os sintomas diminuem em cerca de três meses (WERNECK, MIRANDA, JÚNIOR, 2016).

Sendo assim o bjetivo deste trabalho é discutir aspectos clínicos, histopatológicos e protocolo terapêutico de uma reação liquenóide oral associada a restaurações de amálgama, sem presença de recidiva.

\section{RELATO DE CASO}

Paciente, sexo feminino, 32 anos, feoderma, compareceu à Clínica Escola de Odontologia do Centro Universitário CESMAC, queixando-se de dormência e dor em uma lesão na língua, há aproximadamente três meses, sem motivo aparente. Ao exame intrabucal foi observada placa esbranquiçada, com áreas eritematosas, localizada em borda lateral de língua (Figura 1) e em mucosa jugal bilateral (Figura 2) lisa, com limites difusos, de formato irregular e não infiltrativa.

A hipótese diagnóstica foi de reação liquenóide de contato associada ás restaurações de amálgama, dessa forma, foi necessário a realização de uma biópsia do tipo incisional, sob anestesia local com lidocaína a $2 \%$ e epinefrina 1:100000, em borda lateral de língua, em virtude da mesma apresentar a maior parte da lesão, para posterior avaliação histopatológica.

Os cortes histológicos revelam fragmentos de mucosa revestidos por epitélio pavimentoso queratinizado, com áreas de atrofia e discreta acantose (Figura 3). Na camada basal é possível observar áreas focais de degeneração hidrópica associada a exocitose e lâmina própria apresentando infiltrado inflamatório linfocitário ora em faixa, ora difuso mais profundamente distribuído e folículos linfoides (Figura 4). 


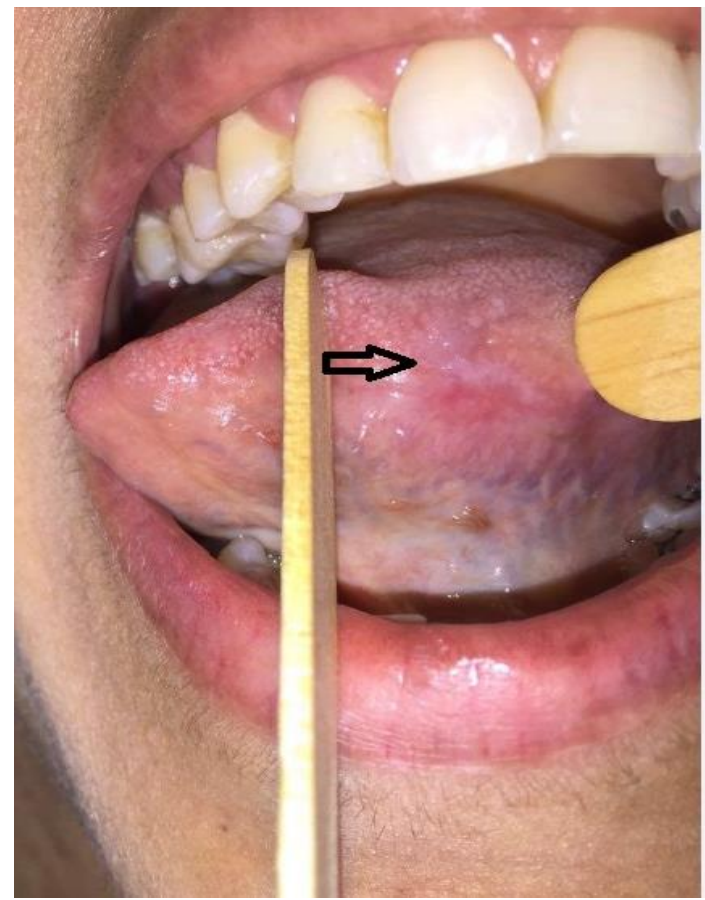

Figura 1: Reação liquenóide em borda lateral de língua

Fonte: Dados da pesquisa.

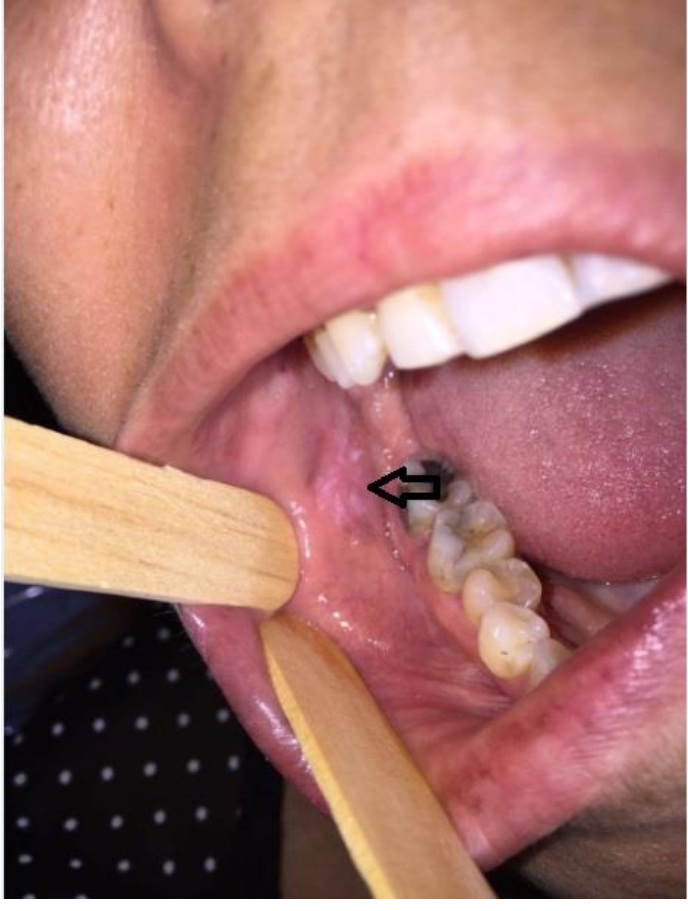

Figura 2: Lesão reacional em mucosa jugal do lado esquerdo

Fonte: Dados da pesquisa

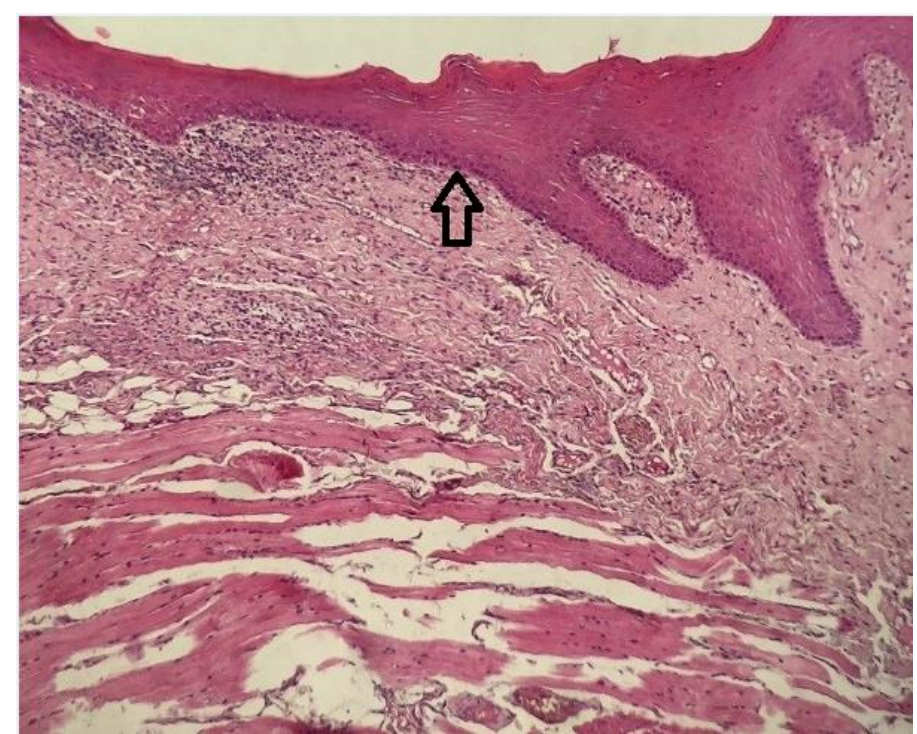

Figura 3: Epitélio queratinizado com discreta acantose Fonte: Dados da pesquisa

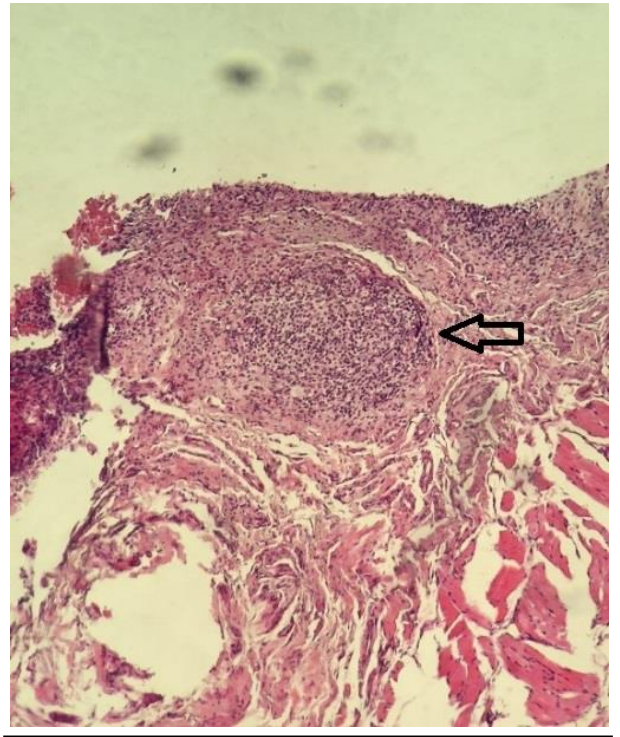

Figura 4: Infiltrado inflamatório linfocitário e folículos linfoides. Fonte: Dados da pesquisa 


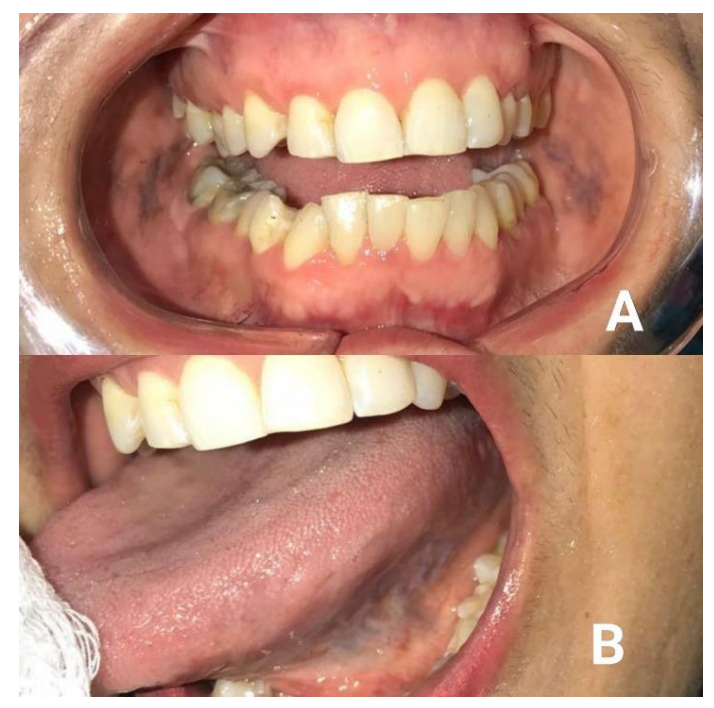

Figura 5.a: Mancha acastanhada em região de mucosa jugal, e B regressão total da lesão. Fonte: Dados da pesquisa
Após confirmação do diagnóstico, foi determinada a substituição das restaurações de amálgama utilizando como material dentário a resina composta, que além de ser estética, possui resistência eficaz em restaurações de dentes posteriores. Para tratamento da sintomatologia, foi prescrito: Clorexidina $0,12 \%$ para uso durante dez dias; Dacktarin gel oral (uso tópico); Admuc gel (pomada); Prednisona (comprimido).

Após dois anos da troca das restaurações, realizou-se o acompanhamento para reavaliar a condição da lesão, as lesões em mucosa jugal lado esquerdo e direito (figura 5.a) regrediram, restando uma mancha de coloração acastanhada no local, de forma assintomática, e a lesão em língua houve total regressão. (figura 5.b)

\section{DISCUSSÃO}

De acordo com a literatura, as reações liquenóides orais tem origem a partir de um episódio de hipersensibilidade associado a materiais odontológicos, medicamentos ou através do contato com materiais metálicos (SANTOS et al., 2017) e apresentam coloração branca. No caso descrito, a lesão manifesta-se através do aparecimento de placas eritematosas esbranquiçadas, que não saem ao raspado e localizam-se próximo a um elemento dentário restaurado com amálgama.

Quanto a sua localização, de acordo com Mendes et al (2015), a reação liquenóide raramente acomete as regiões de palato e língua, discordando assim, de Santos et al (2017) e Barros et al (2015) que descrevem como os principais locais de acometimento da lesão a região posterior da mucosa jugal, superfície ventral das bordas laterais da língua, língua propriamente dita e gengiva.

Neste estudo, relata-se o aparecimento de estrias liquenóides em região de mucosa jugal e borda lateral de língua, corroborando com os estudos previamente citados, que definem as principais localidades de aparecimento das lesões.

Em um estudo realizado por Mendes et al (2015), em que foram analisados 1799 prontuários pertencentes a um laboratório de patologia, aonde 33 apresentavam reação liquenóide oral, 26 eram do gênero feminino e 7 do gênero masculino, evidenciando a predileção da lesão pelo aparecimento em mulheres, assim como Prado et al (1994). Dessa forma, o caso apresentado corrobora com a literatura, sendo o paciente do gênero feminino, na terceira década de vida.

De acordo com Prado et al (1994) as reações liquenóides de contato apresentam-se de forma assintomática, assim como Mendes et al (2015), mas relatam também a possibilidade do aparecimento de sintomas, tais como dor e ardência, em uma pequena parcela da população. Santos et al (2017) descreve ainda a queixa de prurido, desconforto e gosto metálico. Em concordância com a literatura, o paciente do caso relatado, descreve sensação de dormência e dor na região da lesão.

O diagnóstico foi realizado associando os critérios clínicos e histopatológicos da lesão, de forma que foi seguido o que pede na literatura, de acordo com Neto et al, 2013. Histologicamente foi observada a presença de fragmentos de mucosa revestidos por epitélio pavimentoso queratinizado, com áreas de atrofia 
e discreta acantose e infiltrado infamatório. Para tratamento da sintomatologia dolorosa foi prescrito o uso de Clorexidina 0,12\% durante dez dias; Dacktarin gel oral (uso tópico); Admuc gel (pomada); Prednisona (comprimido). E após o tratamento sintomatológico, foi realizada a troca das restaurações de amálgama pela resina composta como material restaurador, pois além de possuir resistência é um material estético e com menor probabilidade de promover reações de hipersensibilidade.

Após o tratamento da lesão, foi realizado o acompanhamento clínico e constatado a ausência de recidiva das estrias, sendo coerente com o estudo de Werneck, Miranda, Júnior (2016), em que falam que os sinais da lesão desaparecem após 3 meses e não apresentam possibilidade de regressão.

\section{CONSIDERAÇÕES FINAIS}

Entre as lesões liquenóides orais, a reação liquenóide de contato e o líquen plano oral formam um desafo ao cirurgião-dentista, em virtude de suas semelhanças. Dessa forma, faz-se necessário o conhecimento das características clínicas e das lesões liquenóides orais, para realização de um diagnóstico preciso e determinação do tratamento a ser realizado, embora ainda exista uma carência de informações quanto a melhor forma de diagnóstico.

\section{REFERÊNCIAS}

1. ÁRTICO, G et al. Reação liquenoide à carbamazepina em mucosa bucal: relato de caso. An Bras Dermatol., São Paulo, v. 86, n. 4 , p.152-155, nov. 2011

2. BERNARDES, VF et al. Lesão liquenóide oral relacionada ao amálgama. An. Bras. Dermatol., Rio de Janeiro , v. 82, n. 6, p. 549552, Dec. 2007

3. BARROS, DR et al. Levantamento de lesões liquenóides orais em um laboratório de patologia bucal do estado de alagoas no período de 2003 a 2013. Incelências: Revistas Cesmac, Maceió, v. 4, n. 1. 2015

4. FERRISSE, TM. Análise das características clínica, histopatológica e imunopatológica das lesões liquenoides orais. Revisão sistemática e estudo prospectivo. 2016. 67 f. Dissertação (Mestrado) - Curso de Pós Graduação em Odontologia, Faculdade de Odontologia de Araraquara, Universidade Estadual Paulista, Araraquara, 2016.

5. LUIZ, AC. Diagnóstico das reações liquenóides de contato envolvendo teste de hipersensibilidade cutânea ao amálgama e tratamento pela substituição do material restaurador. 2007. 64 f. Dissertação (Mestrado) - Curso de Pós Graduação em Odontologia, Faculdade de Odontologia da Usp, São Paulo, 2007

6. MENDES, GG. Estudo imuno-histoquímico da expressão de metalotioneína e proteína p16 em líquen plano e reações liquenóides orais. 2015. 50 f. Dissertação (Mestrado em Ciências Biomédicas) - Universidade Federal de Uberlândia, Uberlândia, 2015.

7. MERCÊS, EC et al. MANIFESTAÇÕES CLÍNICAS OU LESÕES ASSOCIADAS A EFEITOS ADVERSOS A MEDICAMENTOS EM CAVIDADE ORAL. Anais Seminário de Iniciação Científica, Feira de Santana, v. 21, n. 1. out. 2017.

8. NETO, RAA et al. Líquen plano e relação liquenóide: uma discussão diagnóstica. Rev. odontol. UNESP, vol.42, nEspecial, p.0, 2013.

9. PRADO, MCP et al. Reações Liquenóides da mucosa bucal. Rev Odontol Univ São Paulo, São Paulo, v. 8, n. 2, p.221-224, jul/set. 1994.

10. ROZZA, RV et al. Manifestações bucais da doença enxerto contra hospedeiro em pacientes transplantados de medula óssea. Revistas Dens, Maceio, v. 12, n. 2, p.64, nov/abr. 2007.

11. SOARES-SANTOS, KS et al. REAÇÃO LIQUENÓIDE ORAL ASSOCIADA À RESTAURAÇÃO DE AMÁLGAMA: relato de caso. Revista Eletrônica da Academia Brasileira de Odontologia, São Paulo, v. 26, n. 2, p.49-52, fev. 2017. VASCONCELOS, Isabella Maria Bergamo et al. Diagnóstico e tratamento da reação liquenóide de contato. Sci Invest Dent, Maceio, v. 20 (1), n.12. 2015.

12. WERNECK, JT; MIRANDA, FB; SILVA JÚNIOR, A. Desafios na distinção de lesões de Líquen Plano Oral e Reação Liquenóide. Revista Brasileira de Odontologia, Rio de Janeiro, v. 73, n. 3, p.247-252, jul/set. 2016.

13. $5^{\circ}$ Congresso odontológico de Araçatuba, 2015. Líquen plano ou reação liquenóide? Um desafio no processo de anamnese. Araçattuba: Arch Health Invest, 2015. 360 p. 